\title{
Simulação clínica: construção e validação de roteiro para o Suporte Básico de Vida no adulto
}

Clinical simulation: construction and validation of a script for Basic Life Support in adults

Simulación clínica: construcción y validación de un guion de Soporte Vital Básico en adultos

\section{Juliana da Silva Garcia Nascimento, Kleiton Gonçalves do Nascimento"I, Daniela da Silva Garcia Regino"II, Mateus Goulart Alves ${ }^{\mathrm{IV}}$, Jordana Luiza Gouvêa de Oliveirav ${ }^{\mathrm{v}}$, Maria Celia Barcellos Dalri ${ }^{\mathrm{VI}}$}

\begin{abstract}
Resumo: Objetivo: desenvolver e validar um roteiro para planejar e executar a primeira etapa da simulação clínica do Suporte Básico de Vida no adulto em parada cardiorrespiratória: a preparação e suas fases de pré-simulação e pré-briefing/briefing. Método: estudo metodológico realizado por meio de revisão integrativa com uma amostra de sete estudos primários. Após, realizou-se a validação de conteúdo, em junho de 2020, com 16 enfermeiros. Resultados: o roteiro foi composto por pré-simulação e pré-briefinglbriefing e atingiu um Índice de Validade de Conteúdo total de 0,90. Conclusão: desenvolveu-se um roteiro que aborda título, tempo de execução, definição e objetivos da pré-simulação e pré-briefing/briefing, público-alvo, objetivos de aprendizagem, recursos materiais, procedimento e referências, considerado válido em seu conteúdo para planejar e executar a primeira etapa da simulação clínica do Suporte Básico de Vida no adulto, ou seja, a preparação.

Descritores: Enfermeiras e Enfermeiros; Estudantes de Enfermagem; Reanimação Cardiopulmonar; Treinamento por Simulação; Estudo de Validação
\end{abstract}

Abstract: Objective: to develop and validate a script to plan and execute the first stage of the clinical simulation
of Basic Life Support in adults in cardiorespiratory arrest: the preparation and its pre-simulation and pre-briefing/
briefing phases. Method: methodological study performed through an integrative review with a sample of seven
primary studies. Afterwards, content validation was carried out in June 2020 with 16 nurses. Results: the script

IEnfermeira, Mestre, Universidade de São Paulo - Escola de Enfermagem de Ribeirão Preto (EERP), Ribeirão Preto, São Paulo, Brasil. E-mail: mestradounesp28@yahoo.com.br, Orcid: https://orcid.org/0000-0003-1118-2738

IIEnfermeiro, Mestre, Universidade Federal do Triângulo Mineiro (UFTM), Uberaba, Minas Gerias, Brasil. E-mail: kleiton_uniube@yahoo.com.br, Orcid: https://orcid.org/0000-0002-2717-6837

III Enfermeira. Doutora, Universidade Brasil, Fernandópolis, São Paulo, Brasil. E-mail: garciaregino@gmail.com, Orcid: https://orcid.org/0000-0003-0045-7783

${ }^{\mathrm{IV}}$ Enfermeiro. Mestre, Universidade de Franca, Franca, São Paulo, Brasil. E-mail: mateusgoulartalves@gmail.com, Orcid: https://orcid.org/00000003-0680-6817

${ }^{\vee}$ Enfermeira, Especialista, Universidade de São Paulo - Escola de Enfermagem de Ribeirão Preto (EERP), Ribeirão Preto, São Paulo, Brasil. Email: jordana.oliveira@usp.br, Orcid: https://orcid.org/0000-0001-5905-8993

${ }^{V I}$ Enfermeira, Doutora, Universidade de São Paulo - Escola de Enfermagem de Ribeirão Preto (EERP), Ribeirão Preto, São Paulo, Brasil. E-mail: macdalri@eerp.usp.br, Orcid: https://orcid.org/0000-0002-8173-8642 
Simulação clínica: construção e validação de roteiro para o Suporte Básico de Vida no adulto | 2

consisted of pre-simulation and pre-briefing/briefing and achieved a total Content Validity Index of 0.90 . Conclusion: a script was developed addressing the title, execution time, definition and objectives of the presimulation and pre-briefing/briefing, target audience, learning objectives, material resources, procedure and references, considered valid in its content to plan and execute the first stage of the clinical simulation of Basic Life Support in adults, that is, its preparation.

Descriptors: Nurses; Students, Nursing; Cardiopulmonary Resuscitation; Simulation Training; Validation Study

Resumen: Objetivo: desarrollar y validar un guion para planificar y ejecutar la primera etapa de la simulación clínica de Soporte Vital Básico en adultos en paro cardiorrespiratorio: la preparación y sus fases de pre-simulación y pre-briefing/briefing. Método: estudio metodológico realizado mediante una revisión integradora con una muestra de siete estudios primarios. Subsiguientemente, se realizó la validación de contenido en junio de 2020 con 16 enfermeros. Resultados: el guion consistió en pre-simulación y pre-briefing/briefing y alcanzó un Índice de Validez de Contenido total de 0.90. Conclusión: se elaboró un guion que aborda el título, tiempo de ejecución, definición y objetivos de la pre-simulación y pre-briefing/briefing, público destinatario, objetivos de aprendizaje, recursos materiales, procedimiento y referencias, considerado válido en su contenido para planificar y ejecutar la primera etapa de la simulación clínica de Soporte Vital Básico en adultos, es decir, su preparación.

Descriptores: Enfermeras y Enfermeros; Estudiantes de Enfermería; Reanimación Cardiopulmonar; Entrenamiento Simulado; Estudio de Validación

\section{Introdução}

Obter a atenção de um estudante de enfermagem ou profissional enfermeiro, durante uma prática educativa, é um desafio para qualquer educador, pois, geralmente, os aprendizes almejam por sua exposição a estratégias de ensino inovadoras e ativas, capazes de aumentar a satisfação e, até mesmo, o prazer em seus esforços educacionais. ${ }^{1}$ Para atender a essa demanda, vem se destacando uma estratégia de ensino e aprendizagem denominada simulação clínica em saúde. ${ }^{2}$ Ela é definida como uma estratégia educacional que expõe os participantes a cenários hipotéticos que imitam a realidade da prática clínica. ${ }^{3}$

Para viabilizar essa estratégia, é fundamental conceituar e compreender suas etapas, denominadas preparação, participação e debriefing. ${ }^{4}$ A etapa de preparação divide-se em duas fases: a pré-simulação, que abrange o preparo do aluno para o tema proposto na simulação clínica, por meio do envio de materiais educativos e também de treinamento das habilidades, e o pré-briefing/briefing, configurado pela interação entre o facilitador e os alunos, imediata à cena, para esclarecer sobre o cenário, objetivos e os papéis de aprendizagem. ${ }^{5}$ 
3 | Nascimento JSG, Nascimento KG, Regino DSG, Alves MG, Oliveira JLG, Dalri MCB

Já a etapa de participação envolve a realização do cenário proposto, e o debriefing caracteriza-se por um processo de discussão/reflexão, realizado durante ou após o cenário, capaz de estabelecer o desenvolvimento de competência clínica. ${ }^{5} \mathrm{~A}$ articulação das três etapas da simulação clínica torna possível o processo de ensino e aprendizagem de temáticas complexas como o Suporte Básico de Vida (SBV) para enfermagem, por estimular o aluno a ser o sujeito ativo de seu aprendizado e experienciar de forma segura situações que ele vivenciará na prática. ${ }^{6}$

Dessa forma, a etapa de preparação da simulação clínica é um mecanismo valioso para o aprofundamento científico e o estudo do SBV, antecipadamente à vivência proposta e para o esclarecimento do participante sobre o cenário de simulação, de forma objetiva, didática e humanizada. ${ }^{6-7}$ Para a formação de enfermeiros e o aperfeiçoamento desses profissionais que já lidam, em sua rotina, com a dinâmica da ressuscitação cardiopulmonar aplicando o SBV, a simulação clínica e sua etapa de preparação contribuem para a qualidade da assistência prestada e a segurança dos pacientes, o que configura uma articulação pedagógica importante diante do processo de ensino e aprendizagem em enfermagem. ${ }^{2,4,6-8}$

As pesquisas científicas ${ }^{4-5,7-8}$ sobre simulação clínica, abordam de maneira superficial a etapa de preparação e suas fases de pré-simulação e pré-briefing/briefing, o que provoca uma confusão conceitual e, consequentemente, sua inadequada utilização no processo de ensino e aprendizagem. ${ }^{8}$ Essa lacuna assume destaque quando se evidencia a ausência de roteiros na literatura, específicos para condução da etapa de preparação na simulação clínica, fator que pode interferir negativamente no desenvolvimento da simulação, ${ }^{9}$ principalmente sobre ressuscitação cardiopulmonar com SBV. A adoção de constructos válidos e adequadamente estruturados para esse fim, embasados em um percurso metodológico confiável, é importante para fomentar a excelência da aprendizagem dessa temática e alcançar a projeção do conhecimento e raciocínio clínico necessários e essenciais para atendimento da parada cardiorrespiratória. ${ }^{10}$ 
Simulação clínica: construção e validação de roteiro para o Suporte Básico de Vida no adulto | 4

Desta forma, tem-se como questão de pesquisa: Quais conteúdos são necessários para compor um roteiro para a etapa de preparação na simulação clínica do SBV em parada cardiorrespiratória? Este estudo teve como objetivo desenvolver e validar um roteiro para planejar e executar a primeira etapa da simulação clínica do SBV no adulto em parada cardiorrespiratória: a preparação e suas fases de pré-simulação e pré-briefing/briefing.

\section{Método}

Trata-se de estudo metodológico, voltado ao processo de elaboração e validação de um roteiro para a etapa de preparação da simulação clínica de um paciente adulto em parada cardiorrespiratória, atendido por meio de SBV, realizado em uma universidade pública de Ribeirão Preto, São Paulo (SP), no período de janeiro a agosto de 2020, voltado a acadêmicos e profissionais de enfermagem.

Adotou-se um referencial teórico-metodológico que segue três conjuntos de procedimentos: teóricos, empíricos e analíticos. ${ }^{11} \mathrm{O}$ procedimento teórico envolveu a identificação das evidências científicas necessárias para elaborar o roteiro desejado, por meio de um compilado de estudos sobre a etapa de preparação da simulação clínica, e com apoio das diretrizes para a ressuscitação cardiopulmonar de 2020 como referencial teórico para os critérios relativos ao SBV no adulto. ${ }^{12} \mathrm{O}$ procedimento empírico envolveu a validação de conteúdo por experts em simulação clínica em enfermagem e em SBV, e o procedimento analítico consistiu na análise dos resultados de validação, por meio da técnica Delphi, de modo a confirmá-la ou refutá-la. ${ }^{11}$ Cada etapa foi apresentada sequencialmente, para facilitar a compreensão do seguimento deste estudo.

$\mathrm{Na}$ etapa de procedimento teórico, executou-se uma revisão integrativa da literatura, na intencionalidade de compilar evidências científicas, capazes de estruturar um arcabouço teórico quanto aos elementos que viabilizam a etapa de preparação e suas fases de pré-simulação e prébriefing/briefing. Embasou-se no cumprimento das seguintes fases: identificação do tema e 
5 | Nascimento JSG, Nascimento KG, Regino DSG, Alves MG, Oliveira JLG, Dalri MCB

questão norteadora; busca e seleção dos estudos na literatura; categorização; análise dos estudos selecionados e apresentação da revisão. ${ }^{13}$

A pergunta de pesquisa da revisão, foi formulada por meio da estratégia PICO, ${ }^{14}$ configurou-se da seguinte forma: Quais as evidências científicas disponíveis na literatura sobre os conteúdos necessários para compor um roteiro acerca da etapa de preparação da simulação clínica, para possibilitar o desenvolvimento de competência em estudantes e profissionais de enfermagem? A população - "P, caracterizou-se por estudantes e profissionais de enfermagem; a intervenção - "I", abordou a identificação dos elementos que compõem as fases de présimulação e pré-briefing/briefing, e o desfecho - "O" correspondeu ao desenvolvimento de competência clínica em enfermagem.

A busca dos achados foi compreendida em abril de 2020, nas fontes de informação: PubMed, Literatura Latino-Americana e do Caribe em Ciências da Saúde (LILACS), Scopus, Cumulative Index to Nursing and Allied Health Literature(CINAHL) e Web of Science, como visualizado no Quadro 1.

Quadro 1 - Apresentação das fontes de informação, descritores, palavra-chave e estratégias de busca adotadas para a revisão integrativa de literatura. Ribeirão Preto (SP), Brasil, 2020.

\begin{tabular}{|c|c|}
\hline $\begin{array}{c}\text { Fonte de informação, descritores e } \\
\text { palavra-chave }\end{array}$ & Estratégia de busca \\
\hline $\begin{array}{l}\text { PubMed" e Scopus } \\
\text { MeSH*: “Nursing”, "Students, Nursing” } \\
\text { e "Simulation" } \\
\text { Palavras-chave: "pré-simulação", "pré- } \\
\text { briefing" e "briefing" }\end{array}$ & 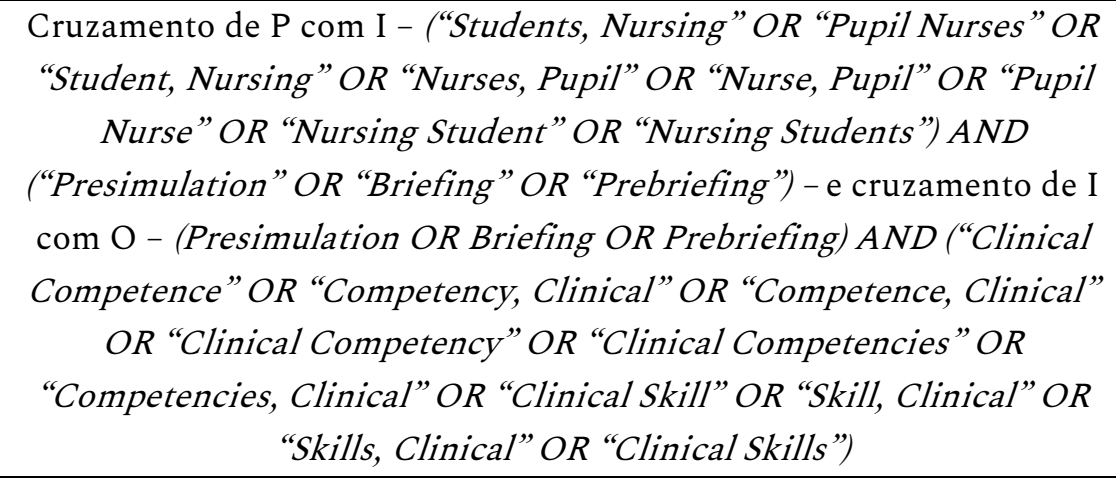 \\
\hline $\begin{array}{l}\text { CINAHL" } \\
\text { Títulos/Assunto: "Students, Nursing”e } \\
\text { "Clinical Competence } \\
\text { Palavras-chave: "Pré-simulation", "Pré- } \\
\text { briefing" e "Briefing” }\end{array}$ & $\begin{array}{l}\text { Cruzamento de P com I ("Students, Nursing” OR "Students, Nurse } \\
\text { Midwifery" OR "Students, Nursing, Associate") AND ("Pré-simulation" } \\
\text { OR "Briefing” OR "Prebriefing”) e cruzamento I com O (Pré-simulation } \\
\text { OR Briefing OR Pré-briefing) AND (“Clinical Competence” OR } \\
\text { "Nursing Skills" OR “Cultural Competence" OR "National Vocational }\end{array}$ \\
\hline
\end{tabular}


Simulação clínica: construção e validação de roteiro para o Suporte Básico de Vida no adulto 6

\begin{tabular}{|c|c|}
\hline & Qualifications") \\
\hline LILACS $^{*}$ & Determinaram-se as seguintes estratégias de busca: cruzamento de $\mathrm{P}$ \\
\hline DeCS $S^{\varsigma}$ Estudantes de Enfermagem”, & com I - (“Students, Nursing” OR “Estudiantes de Enfermería” OR \\
\hline "Equipe de Enfermagem"; & "Estudantes de Enfermagem" AND Pré-simulation OR Pré-simulation \\
\hline "Competência, clínica" & OR Pré-simulação OR Pré-briefing OR Briefing) - e cruzamento I com \\
\hline Palavras-chave: "Pré-simulação", "Pré- & O - (Pré-simulation OR Pré-simulation OR Pré-simulação OR Pré- \\
\hline briefing"e "Briefing & briefing OR Briefing AND "Clinical Competence" OR "Competência \\
\hline (e suas versões em inglês e espanhol) & Clínica” OR “Competência Clínica”. \\
\hline Web of Science & Cruzamento de P com I - (“Students, Nursing” OR “Pupil Nurses” OR \\
\hline Nursing", "Students, Nursing”e & "Student, Nursing” OR "Nurses, Pupil” OR "Nurse, Pupil” OR "Pupil \\
\hline "Simulation" & Nurse" OR "Nursing Student” OR "Nursing Students") AND \\
\hline Palavras-chave: pré-simulação”, “pré- & ("Presimulation" OR "Briefing" OR "Prebriefing") - e cruzamento I com \\
\hline briefing" e "briefing", & O - (Presimulation OR Briefing OR Prebriefing) AND (“Clinical \\
\hline & Competence” OR “Competency, Clinical” OR "Competence, Clinical” \\
\hline & OR "Clinical Competency" OR "Clinical Competencies" OR \\
\hline & $\begin{array}{c}\text { "Competencies, Clinical” OR "Clinical Skill” OR "Skill, Clinical” OR } \\
\text { "Skills Clinical” OR "Clinical Skills") }\end{array}$ \\
\hline & "Skills, Clinical" OR "Clinical Skills"). \\
\hline
\end{tabular}

"MeSH: Medical Subjects Headings; 'CINAHL: Cumulative Index to Nursing and Allied Health Literature, "LILACS: Literatura Latino-Americana e do Caribe em Ciências da Saúde; ${ }^{\S}$ DeCS: Descritores de Ciências da Saúde.

Adotaram-se os operadores booleanos representados pelos termos conectores $A N D$ e $O R$, que permitiram realizar combinações dos descritores utilizados na busca, sendo $A N D$ uma combinação restritiva e $O R$ uma combinação aditiva. ${ }^{14}$ As palavras-chave "pré-simulação", "prébriefing" e "briefing", foram utilizadas a fim de alinhar a estratégia de busca para o objeto pretendido.

Incluíram-se estudos primários, publicados de janeiro de 2009 a abril de 2020. O recorte temporal justifica-se pelo incremento do uso da simulação clínica em enfermagem nesse período e pelo avanço na pesquisa científica sobre as fases da simulação a partir de $2009^{2,8-9}$ sem delimitação do idioma, publicados em periódicos científicos. Revisões da literatura, editoriais, resenhas, relatos de experiências, estudos de caso, reflexões teóricas, dissertações, teses, monografias e resumos publicados em anais de eventos foram excluídos.

Foram realizadas três fases para seleção dos estudos. A primeira abordou a avaliação de títulos e resumos, tendo sido feita por dois profissionais de forma independente, especialistas nas 
7 | Nascimento JSG, Nascimento KG, Regino DSG, Alves MG, Oliveira JLG, Dalri MCB

temáticas propostas, por meio de um programa de revisão gratuito da web de versão única, chamado Rayyan Qatar Computing Research Institute (Rayyan QCRI). Ele auxilia autores de revisões de literatura a realizarem seu trabalho de maneira rápida e permite a exportação dos estudos de uma base de dados determinada para o programa e a exposição de títulos e resumos, com o cegamento do pesquisador auxiliar, o que garante fidedignidade e precisão metodológica. ${ }^{15}$

$\mathrm{Na}$ segunda fase, 15 artigos que ocasionaram divergência entre os pesquisadores foram encaminhados a um terceiro, responsável por tomar a decisão de inclusão ou exclusão. Realizaram-se, na terceira fase, a leitura e a avaliação dos textos completos, para definir a amostra final. Para coleta dos dados, adaptou-se um instrumento previamente validado, ${ }^{16}$ priorizando-se os critérios autores, origem, idioma e ano da publicação, objetivos, delineamento metodológico, resultados e conclusão. Também se classificou o nível de evidência dos estudos. ${ }^{17}$

Os achados foram analisados por meio dos pressupostos da análise temática cumprindose três etapas: a pré-análise, configurada pela leitura flutuante das evidências e organização das informações convergentes, denominadas como unidades de registro; em seguida, a exploração do material com o agrupamento minucioso das unidades de registro identificadas; e o tratamento dos dados, determinando-se as categorias. ${ }^{18}$ Dessa forma, elaborou-se uma categoria denominada: "elementos que compõem as fases de pré-simulação e pré-briefing/briefing na simulação clínica em enfermagem”. Com base nos pressupostos obtidos, elaborou-se o roteiro pretendido. A seleção dos estudos está descrita na Figura $1 .^{19}$ 
Simulação clínica: construção e validação de roteiro para o Suporte Básico de Vida no adulto | 8

Figura 1 - Fluxograma de identificação, seleção e inclusão dos estudos, elaborado a partir da recomendação Preferred Reporting Items for Systematic Reviews and Meta-Analyses (PRISMA). Ribeirão Preto (SP), Brasil, 2020. 
9 | Nascimento JSG, Nascimento KG, Regino DSG, Alves MG, Oliveira JLG, Dalri MCB

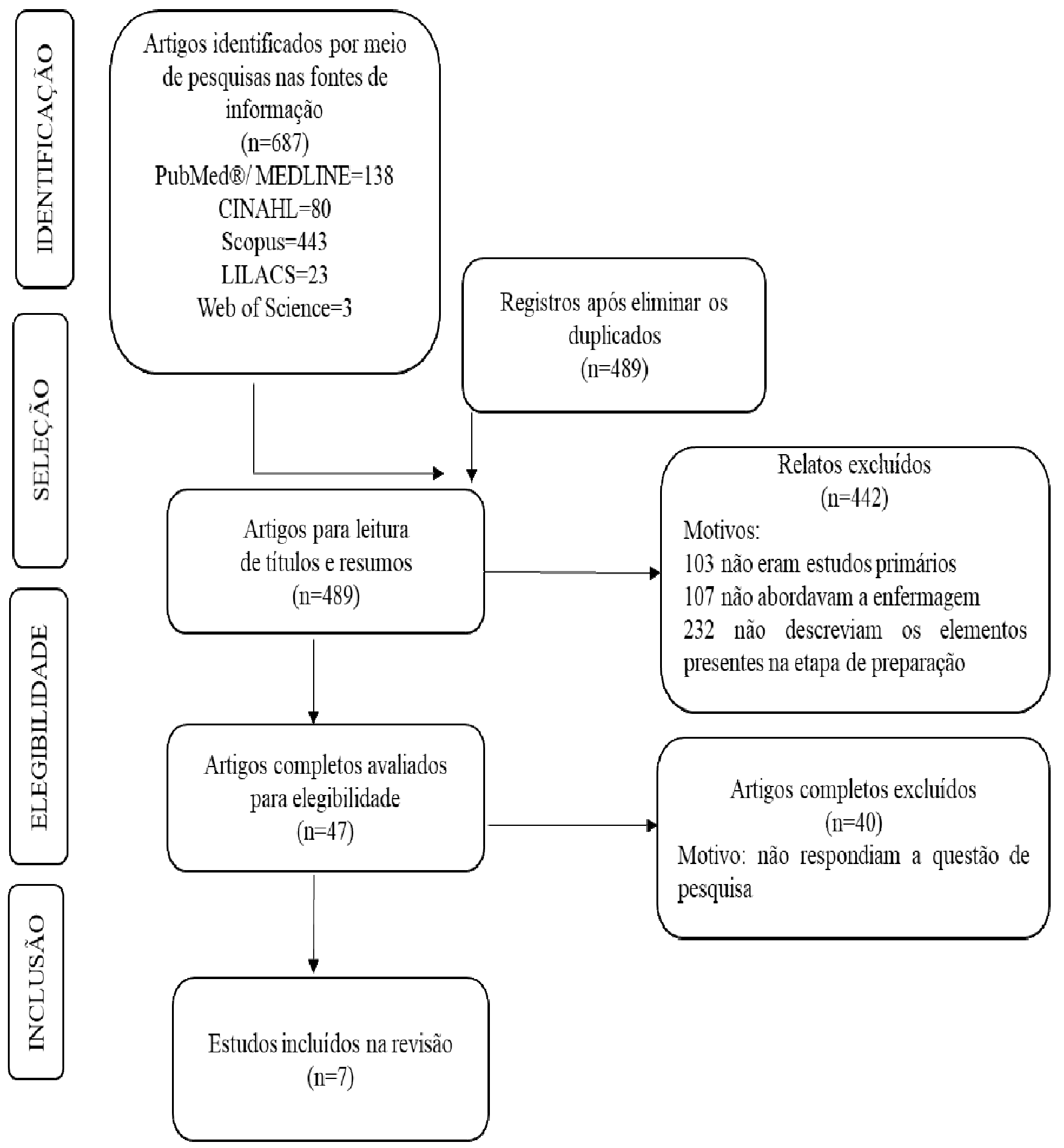

"CINAHL: Cumulative Index to Nursing and Allied Health Literature; ${ }^{\dagger}$ LILACS: Literatura Latino-Americana e do Caribe em Ciências da Saúde.

$\mathrm{Na}$ etapa de procedimentos empíricos, abordou-se o processo de validação de conteúdo do roteiro, realizada em junho de 2020, adotando-se a técnica Delphi. ${ }^{11,20}$ Primeiramente, selecionaram- 
Simulação clínica: construção e validação de roteiro para o Suporte Básico de Vida no adulto | 10

se os experts por meio da análise de seus currículos na Plataforma Lattes, aplicando a seguinte estratégia de busca: no item modo de busca - busca por assunto - simulação em enfermagem; no item bases - doutores; no item formação acadêmica/titulação - Doutorado; no item atuação profissional, grande área - ciências da saúde e enfermagem como área.

Os currículos identificados foram analisados segundo os critérios específicos para o cálculo de escore, que considera quatro pontos para a titulação de Doutor com tese na área de interesse do estudo; três pontos para a titulação de Doutor; três pontos para a titulação de Mestre com dissertação na área de interesse do estudo; dois pontos para a titulação de Mestre; dois pontos para a publicação de artigo em periódico de referência na área de interesse do estudo e dois pontos para a experiência profissional de, no mínimo, 2 anos na área de interesse. Estabeleceu-se valor mínimo de 5 pontos para seleção dos experts na área do construto. ${ }^{21}$

Avaliaram-se, inicialmente, os currículos de 29 experts, visto a possibilidade de perdas devido à não resposta. Destes, 20 obtiveram uma maior classificação de dez pontos, no entanto, 16 profissionais enfermeiros aceitaram participar. Estes foram contactados pela pesquisadora, por e-mail, identificados a partir de seus próprios currículos, em sites de instituições nas quais trabalham ou em artigos publicados. Enviou-se um guia explicativo sobre a pesquisa, os objetivos e Termo de Consentimento Livre e Esclarecido.

O instrumento de coleta de dados foi construído a partir da ferramenta eletrônica gratuita Google Forms, com prazo de 30 dias para resposta, sendo composto de três partes distintas: caracterização dos juízes/experts; análise dos itens que compuseram o roteiro, a partir de uma escala de uma escala do tipo Likert com as opções concordo fortemente (4), concordo (3), não sei (0), discordo (2) e discordo fortemente (1), com espaço aberto para "comentários ou sugestões diante de inadequações”, e critérios gerais de avaliação de conteúdo, segundo os 12 critérios descritos no Quadro $2 .^{11}$ 
11 | Nascimento JSG, Nascimento KG, Regino DSG, Alves MG, Oliveira JLG, Dalri MCB

Quadro 2 - Requisitos de avaliação de conteúdo. Ribeirão Preto (SP), Brasil, 2020.

\begin{tabular}{|c|c|}
\hline Critério & Requisitos \\
\hline Comportamental & O instrumento é aplicável, com instruções claras e exequíveis \\
\hline Objetividade & As recomendações permitem que se alcance o objetivo desejado \\
\hline Simplicidade & Os itens expressam uma única ideia e permitem compreensão adequada \\
\hline Clareza & O conteúdo é explicitado de forma clara e inequívoca \\
\hline Relevância & O instrumento é relevante e atende à finalidade proposta \\
\hline Precisão & Cada item do instrumento é distinto dos demais; eles não se confundem \\
\hline Variedade & A linguagem é adequada e permite interatividade do conteúdo \\
\hline Modalidade & O vocabulário é adequado, sem gerar ambiguidades \\
\hline Tipicidade & O vocabulário é condizente com a temática, com conceitos adequados \\
\hline Credibilidade & A formulação do instrumento contribui com uma atitude favorável de utilização e \\
& \\
\hline Amplitude & O conpreensão do conteúdo \\
\hline Equilíbrio & A sequé atual e consistente, com profundidade suficiente para a compreensão do tema \\
\hline
\end{tabular}

Fonte: Pasquali. ${ }^{11}$

$\mathrm{Na}$ etapa procedimentos analíticos, os achados referentes à validação dos experts foram organizados em planilhas no programa Microsoft Excel 2010, com dupla digitação por dois pesquisadores distintos, para fidedignidade de registro. A análise referente à caracterização dos experts deu-se por meio de estatística descritiva, frequência, percentagem e média. Já as medidas empregadas para avaliar a concordância interavaliadores foram o Índice de Validade de Conteúdo (IVC) por item e o IVC total do instrumento. ${ }^{11,22}$

$A$ priori, empregou-se uma escala do tipo Likert com pontuação de 1 a 4 , que avaliou a relevância/representatividade das respostas dos juízes, a saber: (1) não relevante ou não representativo (discordo fortemente), (2) item necessita de grande revisão para ser representativo (discordo), (3) item necessita de pequena revisão para ser representativo (concordo) e (4) item relevante ou representativo (concordo fortemente). A resposta "não sei” foi considerada como valor zero.

Após, procedeu-se à avaliação do IVC, primeiramente por item do roteiro, adotando-se a fórmula: número de respostas 3 ou 4/número total de respostas obtidas. ${ }^{22}$ Os itens que receberam pontuação 1 ou 2 foram revisados, e as respostas assinaladas como "não sei” foram eliminadas. Em 
Simulação clínica: construção e validação de roteiro para o Suporte Básico de Vida no adulto | 12

seguida, calculou-se o IVC total do instrumento, somando-se cada valor de IVC e dividindo o resultado pelo número de itens que compuseram o roteiro. ${ }^{21}$ Interpretou-se o IVC total do roteiro adotando-se a classificação: resultado $<0,00$ correspondeu à pobre concordância; de 0,00 a 0,20, à leve concordância; de 0,21 a 0,40, à aceitável concordância; de 0,41 a 0,60, à moderada concordância; de 0,61 a 0,80, à considerável concordância e de 0,81 a 1,00, à quase perfeita concordância. Para a presente pesquisa, estabeleceu-se IVC total de 0,80 para indicar o constructo proposto como válido. ${ }^{23}$

As sugestões dos juízes foram avaliadas quanto à sua pertinência, tendo o roteiro sido modificado. Para isso, adotou-se a técnica Delphi, caracterizada pela análise inicial de um questionário/instrumento pelo grupo respondente e de sua concordância. ${ }^{20}$ Cabe ressaltar que, na primeira rodada da técnica Delphi, os itens do roteiro foram considerados válidos, com concordância $>80 \%$ e IVC total acima do esperado $(>0,80) .{ }^{23}$ No entanto, mesmo que os índices de adequabilidade tenham sido atingidos a priori, realizou-se uma segunda rodada da técnica Delphi, para proceder à devolutiva necessária aos juízes, quanto às alterações solicitadas. A pesquisa foi conduzida de acordo com os padrões éticos exigidos pelas resoluções 466/2012, 510/2016 e 580/2018, do Ministério da Saúde, e apresenta o número do protocolo de aprovação 3.826.306, em 6 de fevereiro de 2020.

\section{Resultados}

Compuseram a amostra da presente revisão integrativa sete estudos ${ }^{24-30}$ primários caracterizados por publicações que datam de 2014 a 2018, internacionais, com nível de evidência $2^{17}$ e do tipo ensaio clínico randomizado.

A análise da categoria intitulada "elementos que compõem as fases de pré-simulação e pré-briefing/briefing na simulação clínica em enfermagem” possibitou a construção de um roteiro que torna possível o planejamento e a execução rigorosa da primeira etapa da simulação clínica (a preparação) para o SBV no adulto, o qual dividiu-se em duas partes. A primeira 
13 | Nascimento JSG, Nascimento KG, Regino DSG, Alves MG, Oliveira JLG, Dalri MCB

abordou a pré-simulação e a segunda, o pré-briefing/briefing. Ambas as partes apresentaram os critérios elementos, ações, tempo de execução, definição e objetivos das fases; público-alvo a que a simulação se direciona; os objetivos de aprendizagem para o SBV; os recursos materiais necessários e o procedimento, isto é, um passo-a passo para executar as fases de pré-simulação e pré-briefing, para ser seguido por docentes e facilitadores de simulação clínica em enfermagem.

Do total de 16 (100\%) experts, todos eram enfermeiros especialistas na área da simulação clínica e na ressuscitação cardiopulmonar. A maioria era representada pelo sexo feminino (68,8\%), com idade média de 39 anos e tempo de experiência profissional em enfermagem de, em média, 17 anos. A maioria (14 juízes; 87,5\%) possuía doutorado e atuava em docência no Ensino Superior, com treinamento em simulação, artigos publicados nesse âmbito e já tinham participado de eventos sobre simulação (15 juízes; 93,8\%). Todos os juízes (16; 100,0\%) planejaram e desenvolveram simulações como estratégia de ensino e aprendizagem em enfermagem e dominavam a temática da ressuscitação cardiopulmonar com SBV. A concordância interavaliadores foi evidenciada quanto aos itens que compuseram o roteiro proposto e aos 12 critérios de avaliação de conteúdo ${ }^{11}$ apresentados na Tabela 1.

Tabela 1 - Distribuição das respostas dos experts (16), Índice de Validade de Conteúdo por item e Índice de Validade de Conteúdo total do roteiro sobre a etapa de preparação da simulação clínica no Suporte Básico de Vida. Ribeirão Preto (SP), Brasil, 2020

\begin{tabular}{|c|c|c|c|c|c|c|c|}
\hline \multirow{3}{*}{ Itens do roteiro } & \multicolumn{4}{|c|}{ Relevância da resposta } & \multicolumn{2}{|c|}{ Respostas 3 e 4} & \multirow{3}{*}{$\frac{\text { IVC }^{*}}{(\%)}$} \\
\hline & 0 & 1 & 2 & 3 & 4 & & \\
\hline & $\mathrm{N}(\%)$ & $\mathrm{N}(\%)$ & $\mathrm{N}(\%)$ & $\mathrm{N}(\%)$ & $\mathrm{N}(\%)$ & $\mathrm{IN}(\%)$ & \\
\hline \multicolumn{8}{|l|}{ Fase de pré-simulação } \\
\hline Título & & & $1(6,2)$ & $2(12,5)$ & $13(81,2)$ & $15(93,7)$ & 0,93 \\
\hline Explicação e definição & & $1(6,2)$ & & $2(12,5)$ & $13(81,2)$ & $15(93,7)$ & 0,93 \\
\hline Objetivos & & $1(6,2)$ & & $6(37,5)$ & $9(56,2)$ & $15(93,7)$ & 0,93 \\
\hline Recursos materiais & $1(6,2)$ & & & $5(31,2)$ & $10(62,5)$ & $15(93,7)$ & 0,93 \\
\hline Procedimento & & & $2(12,5)$ & $4(25)$ & $10(62,5)$ & $14(87,5)$ & 0,87 \\
\hline Tempo & & & $2(12,5)$ & $5(31,2)$ & $9(56,2)$ & $14(87,5)$ & 0,87 \\
\hline \multicolumn{8}{|c|}{ Fase de pré-briefing/briefing } \\
\hline Explicação e definição & & & $1(6,2)$ & $5(31,2)$ & $10(62,5)$ & $15(93,7)$ & 0,93 \\
\hline Objetivos & & & $2(12,5)$ & $6(37,5)$ & $8(50,0)$ & $14(87,5)$ & 0,87 \\
\hline
\end{tabular}


Simulação clínica: construção e validação de roteiro para o Suporte Básico de Vida no adulto | 14

\begin{tabular}{|c|c|c|c|c|c|c|c|}
\hline Recursos materiais & & $1(6,2)$ & $1(6,2)$ & $4(25)$ & $10(62,5)$ & $14(87,5)$ & 0,87 \\
\hline Procedimento & $1(6,2)$ & $1(6,2)$ & $2(12,5)$ & $5(31,2)$ & $7(43,7)$ & $12(75,0)$ & 0,75 \\
\hline Tempo & & & $2(12,5)$ & $4(25)$ & $10(62,5)$ & $14(87,7)$ & 0,87 \\
\hline Referências & & & $2(12,5)$ & $2(12,5)$ & $12(75,0)$ & $14(87,5)$ & 0,87 \\
\hline \multicolumn{8}{|l|}{ Critérios gerais de avaliação } \\
\hline Comportamental: é aplicável e claro & & & $2(12,5)$ & $3(18,75)$ & $11(68,7)$ & $14(87,5)$ & 0,87 \\
\hline Objetividade: alcança o objetivo & & & & $5(31,2)$ & $11(68,7)$ & $16(100,0)$ & 1,00 \\
\hline Simplicidade: expressa uma ideia & & & $2(12,5)$ & $5(31,2)$ & $9(56,2)$ & $14(87,5)$ & 0,87 \\
\hline Clareza: o conteúdo é claro & & & $2(12,5)$ & $4(25)$ & $10(62,5)$ & $14(87,5)$ & 0,87 \\
\hline Relevância: o roteiro é relevante & & & & $7(43,7)$ & $9(56,2)$ & $16(100,0)$ & 1,00 \\
\hline Precisão: cada item é distinto & & & $2(12,5)$ & $5(31,2)$ & $9(56,2)$ & $14(87,5)$ & 0,87 \\
\hline Variedade: a linguagem é adequada & & & $1(6,2)$ & $6(37,5)$ & $9(56,2)$ & $15(93,7)$ & 0,93 \\
\hline Modalidade: vocabulário é adequado & $1(6,2)$ & & & $7(43,7)$ & $8(50,0)$ & $15(93,7)$ & 0,93 \\
\hline Tipicidade: o vocabulário é condizente & & & & $8(50,0)$ & $8(50,0)$ & $16(100,0)$ & 1,00 \\
\hline Credibilidade: é compreensível & & & & $8(50,0)$ & $8(50,0)$ & $16(100,0)$ & 1,00 \\
\hline Amplitude: é atual & & & $1(6,2)$ & $7(43,7)$ & $8(50,0)$ & $15(93,7)$ & 0,93 \\
\hline Equilíbrio & & & $2(12,5)$ & $6(37,5)$ & $8(50,0)$ & $14(87,5)$ & 0,87 \\
\hline IVC total & & & & & & & 0,90 \\
\hline
\end{tabular}

"IVC: Índice de Validade de Conteúdo por item; IVC total: Índice de Validade de Conteúdo geral do roteiro

Quanto à concordância interavaliadores, analisada primeiramente para cada item do roteiro proposto, todos os critérios avaliados foram considerados como "quase perfeita concordância” $(0,81$ a 1,00$)$, exceto o décimo item do roteiro, referente à fase de prébriefinglbriefing, denominado Procedimento e classificado como considerável concordância $(0,75)$. Mesmo obtendo um valor acima de 0,80 na maioria dos itens, aqueles cuja avaliação resultou em "discordo" ou "discordo fortemente" foram revisados.

Obteve-se o valor de 0,90 (quase perfeita concordância) para o IVC total do roteiro, o que subsidiou caracterizá-lo como um constructo válido em seu conteúdo, para conduzir a etapa de preparação, na simulação clínica do SBV no adulto. Em geral, as sugestões, os comentários e os apontamentos realizados pelos experts foram avaliados e considerados para elaboração do roteiro, aperfeiçoando, principalmente, os seguintes critérios: título, público-alvo, objetivos de aprendizagem para o SBV, recursos necessários, procedimento e referências.

Dessa forma, após a validação, o roteiro foi constituído por 12 conteúdos: (1) título: o roteiro foi intitulado: "Simulação clínica do Suporte Básico de Vida: roteiro para a etapa de 
15 | Nascimento JSG, Nascimento KG, Regino DSG, Alves MG, Oliveira JLG, Dalri MCB

preparação” (Anexo A); (2) tempo: estabeleceu-se o tempo indicado para planejamento e execução das fases de pré-simulação e pré-briefing/briefing; e (3) referências. Esses três itens iniciais foram comuns a ambas as partes do roteiro. Após, estabeleceram-se cinco conteúdos pertinentes à fase de pré-simulação: (4) definição da fase de pré-simulação para evitar a confusão conceitual entre pré-simulação e pré-briefing/briefing, e os objetivos da fase de pré-simulação; (5) público-alvo para utilização do roteiro; (6) objetivos de aprendizagem do SBV; (7) recursos necessários para planejar a pré-simulação; (8) procedimento: um passo a passo para executar com praticidade, organização e eficiência a fase de pré-simulação. Em seguida, estabeleceramse quatro conteúdos para a fase de pré-briefing/briefing: (9) definição dessa fase e objetivos; (10) público-alvo; (11) recursos necessários; (12) procedimento: um passo a passo para guiar o planejamento e a execução do pré-briefing/briefing.

\section{Discussão}

Nas diversas áreas da educação no âmbito da saúde, pode-se perceber um número crescente de instrumentos que buscam avaliar e conduzir um fenômeno, o que ressalta a importância de sua validade, na tentativa de minimizar julgamentos subjetivos. ${ }^{23}$ No entanto, referente à simulação clínica em enfermagem e, especialmente, ao planejamento de suas etapas, a construção e a validação de instrumentos, é incipiente..$^{7-9}$

Baseando-se nessa fragilidade da literatura científica quanto ao tema proposto na presente pesquisa, faz-se importante ressaltar que os estudos que viabilizaram o desenvolvimento do roteiro para etapa de preparação na simulação clínica do SBV apresentaram alto nível de evidência, configurados em sua totalidade por ensaios clínicos randomizados. Um estudo experimental permite ao pesquisador identificar relações de causa e efeito, e controlar variáveis intrínsecas e extrínsecas, que possam ameaçar a validade interna dos achados, provocando vieses que comprometem os resultados. $^{16}$ Para a enfermagem, fundamentar o desenvolvimento de 
Simulação clínica: construção e validação de roteiro para o Suporte Básico de Vida no adulto | 16 instrumentos, baseando-se em evidências de alta qualidade, é um aspecto significativo, pois fornece uma documentação que permite manter ou alterar a prática atual, de forma segura e consistente. ${ }^{17}$

A adequação do roteiro proposto, baseando-se em critérios bem fundamentados é uma etapa importante do desenvolvimento deste constructo, por torná-lo capaz de determinar se o seu conteúdo explora, de maneira efetiva, os quesitos necessários para alcance de seu objetivo. ${ }^{23}$ Corrobora com essa afirmação estudo que pretendeu desenvolver e validar o conteúdo de um questionário on-line para avaliação do risco de quedas em idosos e valorizou critérios de avaliação como clareza e pertinência, o que tornou possível considerar o instrumento válido em seu conteúdo e adequado para utilização. ${ }^{23}$

O roteiro desenvolvido traz como maior potencialidade a articulação da simulação clínica, como estratégia pedagógica inovadora, ativa e eficaz para o processo de ensino e aprendizagem em enfermagem, com a temática do SBV no adulto. Ainda apresenta como benefícios a padronização e a organização dos conteúdos necessários para o planejamento e a execução da etapa de preparação da simulação clínica, de maneira que possa ser adaptada para outros temas no ensino em enfermagem, o que torna esse instrumento versátil, útil e de fácil manipulação.

Pelo fato de a simulação clínica ser uma estratégia educacional em ascensão tanto no contexto internacional como nacional, podem existir incertezas metodológicas para sua condução, principalmente quanto às fases de pré-simulação e pré-briefing/briefing. ${ }^{4-5}$ Dessa forma, a obtenção de um instrumento validado e baseado em evidências científicas ${ }^{10}$ auxilia seu desenvolvimento e proporciona segurança para o ensino de temáticas complexas, como a ressuscitação cardiopulmonar. ${ }^{6,12,28}$

Os elementos que compuseram o roteiro proposto, quanto às fases da preparação na simulação clínica do SBV, destacaram, inicialmente, a definição das fases e seus objetivos, visto que se faz importante que um facilitador de simulação em enfermagem obtenha compreensão conceitual da pré-simulação e pré-briefing/briefing, para manter o rigor durante sua execução. 
17 | Nascimento JSG, Nascimento KG, Regino DSG, Alves MG, Oliveira JLG, Dalri MCB

Estudos que abordaram as fases da etapa de preparação conceituaram que, enquanto a présimulação é uma fase que viabiliza o envio de referenciais científicos para estudo prévio do participante e o treinamento de habilidades, o pré-briefing/briefing configura o esclarecimento quanto aos elementos que envolvem e interferem no cenário simulado e em seu desempenho.,7-9

Nesse ínterim, ressalta-se que ocorre, na atualidade, uma confusão quanto a esses conceitos, por exemplo, a exclusão da fase de pré-simulação e a realização direta do prébriefinglbriefing na simulação clínica, ou, até mesmo, considerá-las como sinônimos. Isso justifica a necessidade do roteiro da etapa de preparação conter a definição de suas fases e sua intencionalidade, visando à correta diferenciação e utilização.-5,9

Em seguida, os elementos identificados expuseram a importância de apontar o públicoalvo e os objetivos de aprendizagem para o SBV. Esse roteiro é voltado ao processo de ensino e aprendizagem de profissionais enfermeiros e estudantes de graduação em enfermagem e estabelece um roll de objetivos de aprendizagem que devem ser embasados na taxonomia de Bloom e nas diretrizes da American Heart Association (AHA). ${ }^{6}$

Pesquisa experimental randomizada ${ }^{24}$ realizada com 38 enfermeiros norte-americanos verificou se o pré-briefing/briefing é capaz de desenvolver competência clínica para a ressuscitação cardiopulmonar e identificou que o estabelecimento rigoroso dos objetivos de aprendizagem do SBV, durante a etapa de preparação, possibitou o rastreamento dos resultados de aprendizagem, melhorou o trabalho em equipe, a liderança dos indivíduos e a qualidade e rapidez na ressuscitação cardiopulmonar. ${ }^{24}$

Os materiais, instrumentos e as ações que viabilizam a etapa de preparação também foram valorizados pelo roteiro, e, nesta perspectiva, estudos de revisão sistemática ${ }^{4-5}$ fundamentaram a importância de se estabelecerem, principalmente, as ações pertinentes à pré-simulação e ao prébriefing/briefing, no âmbito da educação, visto que a ausência desse elemento pode comprometer os resultados de aprendizagem dos indivíduos submetidos a essa estratégia de ensino. ${ }^{4-5}$ 
Simulação clínica: construção e validação de roteiro para o Suporte Básico de Vida no adulto | 18

A principal intencionalidade da validação do roteiro proposto foi garantir sua efetividade e confiabilidade no planejamento e a execução da primeira etapa da simulação clínica, a preparação para o SBV, visto que a execução adequada da pré-simulação e do prébriefinglbriefing vem sendo associada à redução dos níveis de estresse em estudantes de enfermagem durante a simulação clínica, o que maximiza o processo de ensino e aprendizagem e potencializa o sucesso das etapas posteriores. ${ }^{4-5}$

O processo de validação do roteiro contou com a avaliação crítica de 16 experts em simulação clínica e SBV, além de sua expertise na área e do fator fundamental para se obter um constructo válido e adequado. ${ }^{23}$ Esse número de juízes também foi adotado por um estudo de validação, ${ }^{10}$ que objetivou construir e validar um questionário sobre ressuscitação cardiopulmonar no adulto em SBV, com o uso do desfibrilador externo automático, no ambiente hospitalar, resultando na elaboração de um questionário de 20 questões de múltipla escolha com concordância interavaliadores "quase perfeita" para o assunto abordado. ${ }^{10}$

O IVC total do roteiro, de "quase perfeita concordância", evidencia seu reconhecimento científico e criticidade na avaliação do conteúdo, para o alcance dos objetivos almejados no processo de ensino-aprendizagem do SBV no adulto para a enfermagem. ${ }^{23}$

As principais limitações foram o número incipiente de artigos científicos que abordam a etapa de preparação na simulação clínica em enfermagem e a ausência de estudos que se propuseram a elaborar e validar roteiros para a conduzir essa etapa.

Este estudo é relevante e confere ineditismo à ciência em enfermagem, visto que reúne evidências científicas fidedignas sobre a etapa de preparação da simulação clínica e suas fases de pré-simulação e pré-briefing/briefing, ainda pouco exploradas no âmbito da educação em enfermagem, por meio da simulação clínica. Também oferece um produto científico em formato de roteiro, para o planejamento e a condução da etapa inicial da simulação clínica, que pode ser 
19 | Nascimento JSG, Nascimento KG, Regino DSG, Alves MG, Oliveira JLG, Dalri MCB

facilmente adaptado para diversas outras realidades diante do processo de ensino e aprendizagem em saúde, o que torna esse constructo versátil e útil (Anexo A).

\section{Conclusão}

O roteiro foi desenvolvido a partir da identificação dos conteúdos pertinentes à etapa de preparação da simulação clínica do SBV no adulto, composto por título do roteiro, tempo de execução de cada fase, definição e objetivos das fases de pré-simulação e pré-briefing/briefing, público-alvo, objetivos de aprendizagem do SBV, recursos materiais, procedimento para execução e referências. Considerou-se o roteiro válido em seu conteúdo por atingir Índice de Validade de 0,90, caracterizado como “quase perfeita concordância”.

Este estudo contribui para a pesquisa, a assistência e o ensino em enfermagem, por apresentar um roteiro validado e de fácil manipulação, que propicia e subsidia o planejamento e a execução da primeira etapa de uma simulação clínica, por facilitadores e docentes em enfermagem, diante do processo de ensino e aprendizagem da ressuscitação cardiopulmonar no adulto, baseando-se em evidências científicas atuais e confiáveis, tanto quanto por possibilitar sua adaptação e utilização para a simulação clínica em outras temáticas de ensino e para outras profissões voltadas à saúde, o que favorece a disseminação da ciência e das pesquisas científicas nesse âmbito.

Sugere-se o desenvolvimento de estudos metodologicamente bem delineados, que proponham comparar a efetividade do processo de ensino e aprendizagem, por meio da simulação clínica para o SBV, utilizando o roteiro desenvolvido na presente pesquisa. 
Simulação clínica: construção e validação de roteiro para o Suporte Básico de Vida no adulto 20

\section{Anexo A: Simulação clínica do Suporte Básico de Vida: roteiro para a etapa de preparação}

\begin{tabular}{|c|c|c|}
\hline \multicolumn{3}{|c|}{ Fase de pré-simulação } \\
\hline Elementos & Ações & Tempo \\
\hline $\begin{array}{l}\text { Definição e } \\
\text { objetivos da fase } \\
\text { de pré- } \\
\text { simulação }\end{array}$ & $\begin{array}{l}\text { Definição: A "Preparação" é a primeira etapa da simulação clínica, que se } \\
\text { subdivide nas fases de pré-simulação e pré-briefing/briefing. A pré-simulação } \\
\text { aborda o preparo do participante acerca do tema proposto para a simulação } \\
\text { clínica, por meio de materiais didáticos e treinamento de habilidades. Objetivos: } \\
\text { (1) Orientar com antecipação e de forma planejada o participante da simulação } \\
\text { clínica; (2) Favorecer o desenvolvimento de competência clínica para o SBV } \\
\text { (habilidades cognitivas-conhecimento; habilidades psicomotoras- habilidades } \\
\text { práticas e habilidades afetivas-atitudes). }\end{array}$ & $\begin{array}{c}\text { O tempo } \\
\text { geralmente é } \\
\text { de } 15 \text { dias, que } \\
\text { antecedem a } \\
\text { realização do } \\
\text { cenário de } \\
\text { simulação, no } \\
\text { entanto, pode } \\
\text { ser estendido. }\end{array}$ \\
\hline Público-alvo & $\begin{array}{l}\text { Profissionais enfermeiros e estudantes de graduação em enfermagem, de } \\
\text { preferência, que já tenham tido contato com o ambiente hospitalar e com a } \\
\text { disciplina de bases técnicas. }\end{array}$ & \\
\hline $\begin{array}{l}\text { Objetivos de } \\
\text { aprendizagem } \\
\text { do Suporte } \\
\text { Básico de Vida }\end{array}$ & $\begin{array}{l}\text { Elaborados por meio da Taxonomia de Bloom e das diretrizes da American Heart } \\
\text { Association (2015) para o SBV. Para desenvolver as habilidades cognitivas } \\
\text { (conhecimento) e habilidades psicomotoras (prática): (1) Conhecer e compreender a } \\
\text { cadeia de sobrevivência intra-hospitalar; (2) Analisar a cadeia de sobrevivência } \\
\text { intra-hospitalar articulando-a com a experiência realizada no cenário simulado do } \\
\text { SBV; (3) Conhecer, compreender e sintezar a importância da Vigilância e } \\
\text { Prevenção, como primeiro elo da Cadeia de Sobrevivência Intra-hospitalar; (4) } \\
\text { Analisar e avaliar a importância do reconhecimento imediato da parada } \\
\text { cardiorrespiratória (PCR) e acionamento do Serviço Médico de Emergência; (5) } \\
\text { Compreender e avaliar as características da Ressucitação Cardiopulmonar (RCP) } \\
\text { de alta qualidade com SBV: a frequência ideal da compressão torácica externa } \\
\text { (CTE); profundidade da CTE; permisão do retorno do tórax após a compressão; } \\
\text { minimizar as interrupções entre as compressões; posicionamento ideal das mãos } \\
\text { durante a compressão; evitar ventilação excessiva; relação compressão-ventilação } \\
\text { sem via aéria avançada, utilização correta do Desfibrilador Externo Automático } \\
\text { (DEA). Para desenvolver as habilidades afetivas (atitudes): (1) Ter disposição e } \\
\text { atenção para aprender; (2) Participar ativamente e com satisfação; (3) Estabelecer } \\
\text { compromisso com o aprendizado; (4) Dar valor a cada situação aprendida e } \\
\text { contextualizá-la; (5) Transferir o aprendizado experiencial para a prática real. }\end{array}$ & \\
\hline $\begin{array}{c}\text { Recursos } \\
\text { necessários }\end{array}$ & $\begin{array}{l}\text { Para o estudo prévio acerca do SBV em adulto- enviar por meio eletrônico, os } \\
\text { seguintes materiais didáticos: (1) Videoaula: RCP no adulto em SBV com o uso do } \\
\text { DEA no ambiente } \\
\text { (https://www.youtube.com/watch?v=MT4DJ5sazik\&t=435s); (2) Vídeo de simulação: } \\
\text { RCP no adulto no SBV com o uso do DEA no ambiente hospitalar } \\
\text { (https://www.youtube.com/watch?v=xvmOepMeQd4\&t=62s); (3) Material intitulado } \\
\text { "Destaques da American Heart Association } 2015 \text { - Atualização das Diretrizes de } \\
\text { RCP e ACE - Resumido em Português (https://eccguidelines.heart.org/wp- } \\
\text { content/uploads/2015/10/2015-AHA-Guidelines- Highlights-Portuguese.pdf). } \\
\text { Para o treinamento de habilidades psicomotoras do SBV, em laboratório de }\end{array}$ & $\begin{array}{l}15 \text { dias que } \\
\text { antecedem a } \\
\text { atividade de } \\
\text { simulação } \\
\text { clínica } \\
\text { proposta }\end{array}$ \\
\hline
\end{tabular}




\begin{tabular}{|c|c|c|}
\hline & $\begin{array}{l}\text { habilidades clínicas, ou em ambiente in situ, organizar: (1) Materiais permanentes: } \\
\text { manequim do tipo torso, manequim de média fidelidade com feedback para } \\
\text { frequência e profundidade de compressões torácicas externas, tela de televisão para } \\
\text { captar imagem do software por meio de bluetooth, manequim de alta fidelidade; } \\
\text { carrinho de emergência; escadinha; unidade bolsa-valva-máscara; óculos de proteção; } \\
\text { régua de gases completa com dispositivos acoplados, como umidificador de oxigênio, } \\
\text { contendo água destilada no nível mínimo estimado; sistema de aspiração com frasco; } \\
\text { DEA para treinamentos; maca hospitalar e leito hospitalar; (2) Materiais de consumo: } \\
\text { luvas de procedimento (P, M e G) e máscaras faciais tipo cirúrgica. }\end{array}$ & $\begin{array}{l}\text { preferência, } \\
\text { realizar no } \\
\text { mesmo dia da } \\
\text { execução do } \\
\text { cenário de } \\
\text { simulação } \\
\text { clínica, antes } \\
\text { do cenário }\end{array}$ \\
\hline Procedimento & $\begin{array}{l}\text { Para o estudo prévio acerca do SBV em adulto: (1) Determinar a lista de } \\
\text { participantes e os endereços eletrônicos para os quais os materiais didáticos } \\
\text { selecionados para estudo serão enviados; (2) Enviar, juntamente aos materiais de } \\
\text { estudo determinados, uma apresentação sobre a simulação clínica do SBV no } \\
\text { adulto. Deve ser uma proposta que aborde os objetivos de aprendizagem } \\
\text { pretendidos, as etapas da simulação clínica que serão realizadas, os facilitadores } \\
\text { de simulação clínica que estarão presentes (minicurrículo), a importância da etapa } \\
\text { de preparação e como procedê-la e as datas, horários, local, duração e organização } \\
\text { da simulação clínica a qual o participante será submetido; } \\
\text { Para o treinamento de habilidades psicomotoras do SBV: (1) O treinamento de } \\
\text { habilidades será realizado em grupos de } 5 \text { participantes, dada a maximização do } \\
\text { processo de ensino e aprendizagem em um número reduzido de pessoas, pela } \\
\text { possibilidade de um melhor acompanhamento, mais atenção e melhor avaliação; } \\
\text { (2) Organizar previamente o laboratório de habilidades da instituição em que a } \\
\text { simulação clínica ocorrerá, dispondo de um manequim do tipo torso para cada três } \\
\text { participantes; (3) Receber os participantes no laboratório de habilidades, } \\
\text { apresentar os facilitadores ou equipe de instrutores da simulação proposta e } \\
\text { realizar as devidas orientações sobre como o treinamento de habilidades ocorrerá } \\
\text { e sua importância; (4) Iniciar o treinamento, demonstrando uma videoaula sobre o } \\
\text { SBV, e a cada etapa visualizada do atendimento clínico do SBV com uso do DEA, } \\
\text { paralisar a exposição do vídeo, demonstrar no manequim do tipo torso no chão e } \\
\text { propor a execução pelos participantes; (5) Após, realizar o SBV, utilizando o } \\
\text { manequim de média fidelidade, no leito ou na maca hospitalar, com feedback para } \\
\text { frequência e profundidade de compressões torácicas externas e tela de televisão } \\
\text { para captar imagem do software por meio de bluetooth }\end{array}$ & $\begin{array}{l}15 \text { dias que } \\
\text { antecedem a } \\
\text { atividade } \\
\text { proposta }\end{array}$ \\
\hline \multicolumn{3}{|c|}{ Fases de pré-briefing/briefing } \\
\hline $\begin{array}{c}\text { Definição e } \\
\text { objetivos das } \\
\text { fases de pré- } \\
\text { briefing/briefing }\end{array}$ & $\begin{array}{l}\text { Definição: o pré-briefing/briefing é a fase que envolve a organização e o } \\
\text { esclarecimento dos participantes quanto ao ambiente e ao cenário de simulação } \\
\text { proposto. Objetivos: (1) Oferecer as informações preparatórias para execução da } \\
\text { simulação, como os objetivos do cenário, os papéis a serem desempenhados pelos } \\
\text { participantes e a visão geral dos equipamentos disponíveis; (2) Compartilhar } \\
\text { conhecimento, reduzir a confusão sobre os papéis dos participantes e ajudar a } \\
\text { criar um modelo mental quanto às metas de atendimento ao paciente; (3) Orientar } \\
\text { quanto a ambiente simulado, laboratório e manequins; seu funcionamento; } \\
\text { objetivos do cenário; relatório ou histórico de informações sobre o quadro clínico } \\
\text { do cliente e papéis e responsabilidades específicas dos membros da equipe; (4) }\end{array}$ & $\begin{array}{c}\text { Antecede } \\
\text { imediatamente } \\
\text { o cenário de } \\
\text { simulação } \\
\text { clínica }\end{array}$ \\
\hline
\end{tabular}




\begin{tabular}{|c|c|c|}
\hline & $\begin{array}{l}\text { Obter um melhor trabalho em equipe e desempenho organizado na simulação } \\
\text { clínica. }\end{array}$ & \\
\hline Público-alvo & $\begin{array}{l}\text { Profissionais enfermeiros e estudantes de graduação em enfermagem, de } \\
\text { preferência, que já tenham tido contato com o ambiente hospitalar e com a } \\
\text { disciplina de bases técnicas. }\end{array}$ & \\
\hline $\begin{array}{c}\text { Recursos } \\
\text { necessários }\end{array}$ & $\begin{array}{l}\text { Em laboratório de habilidades clínicas ou em ambiente in situ, organizar os } \\
\text { matérias necessários para a execução do cenário intitulado: "Ressuscitação } \\
\text { cardiopulmonar no adulto em ambiente hospitalar, com Suporte Básico de Vida e } \\
\text { uso do desfibrilador externo automático" } \\
\text { (1) Materiais permanentes: utilizar manequim de alta fidelidade; carrinho de } \\
\text { emergência; escadinha; unidade bolsa-valva-máscara; óculos de proteção; régua de } \\
\text { gases completa, com dispositivos acoplados como umidificador de oxigênio, } \\
\text { contendo água destilada no nível mínimo estimado; sistema de aspiração com } \\
\text { frasco; DEA para treinamentos; leito hospitalar; monitor cardíaco; oxímetro de } \\
\text { pulso; eletrodos para monitorização cardíaca; lençol e travesseiro. (2) Materiais de } \\
\text { consumo: luvas de procedimento (P, M e G) e máscaras faciais do tipo cirúrgica. } \\
\text { Não são necessários atores para execução deste cenário, apenas os participantes } \\
\text { envolvidos na simulação clínica }\end{array}$ & \\
\hline Procedimento & $\begin{array}{l}\text { 1. Estabelecer primeirante o “contrato de ficção” da simulação clínica, que: (1) } \\
\text { Normatiza o compromisso dos facilitadores e participantes da simulação clínica } \\
\text { com a segurança psicológica dos participantes e confidencialidade de todas as } \\
\text { atividades e ocorrências durante a simulação; (2) Esclarece quem orientará e } \\
\text { acompanhará o desempenho dos participantes, e que não se trata de uma avaliação } \\
\text { punitiva; (3) Estabelece o compromisso do participante em fazer a sua parte e agir } \\
\text { como se tudo fosse real, para tirar o máximo proveito dessa experiência; (4) } \\
\text { Esclarece que cada participante terá um papel previamente comunicado e } \\
\text { esclarecido durante a execução do cenário; (5) Informa que será utilizado o exame } \\
\text { clínico objetivo estruturado (OSCE), para registrar o desempenho de cada } \\
\text { participante durante a execução do SBV e que, por isso, a cena será repetida, até } \\
\text { todos os participantes desempenharem todos os papéis abordados por este } \\
\text { instrumento; (6) Explica que, após o término do cenário, haverá o debriefing, que } \\
\text { deve durar o “dobro do tempo”, sendo utilizado para a execução do cenário, } \\
\text { conduzido pelos facilitadores da simulação para reflexão sobre a vivência. } \\
\text { Orientar quanto a: (7) Ambiente de aprendizagem: demonstrar a localização de } \\
\text { todos os materiais utilizados durante a cena, o manequim e os equipamentos; (8) } \\
\text { Objetivos de aprendizagem (dispostos anteriormente); (9) Apresentação do } \\
\text { seguinte caso clínico: “Trata-se de um paciente de } 50 \text { anos, Sr. Alfredo, internado } \\
\text { no leito } 203 \text { da enfermaria de um hospital, com histórico de vômitos, dor } \\
\text { epigástrica e precordial, irradiação para região torácica posterior e diagnóstico } \\
\text { médico de infarto agudo do miocárdio. Encontra-se arresponsivo e arreativo no } \\
\text { leito. Ao realizar a visita à beira leito para avaliação de enfermagem do S.r. } \\
\text { Alfredo, o enfermeiro nota diminuição/ausência de reação e percepção” ; (1)) } \\
\text { Definir o papel de cada partipante na cena: a cena contará com a presença de, no } \\
\text { máximo, cinco participantes; (11) Papéis: Primeiro participante (líder): iniciará a } \\
\text { cena com uma visita à beira do leito, abordará o paciente em questão, perceberá a }\end{array}$ & $\begin{array}{c}\text { Antecede } \\
\text { imediatamente } \\
\text { a execução do } \\
\text { cenário de } \\
\text { simulação, } \\
\text { com duração } \\
\text { de } 10 \text { minutos }\end{array}$ \\
\hline
\end{tabular}




\begin{tabular}{|c|c|}
\hline & $\begin{array}{l}\text { ausência de reatividade e chamará os outros participantes à cena, dividindo suas } \\
\text { funções, Segundo participante: assumirá as compressões torácicas, Terceiro } \\
\text { participante: assumirá a ventilação com bolsa-válvula-máscara, Quarto } \\
\text { participante: assumirá o DEA, Quinto participante: assumirá a substituição das } \\
\text { compressões torácicas externas } \\
\text { Tempo de duração do cenário: o cenário será interrompido para troca de papéis, } \\
\text { após o retorno da circulação espontânea, depois do primeiro choque, e será } \\
\text { finalizado, após todos os participantes desempenharem todos os papéis, Permitir } \\
\text { aos participantes alguns minutos antes do início do cenário, para processarem e } \\
\text { repassarem as informações obtidas }\end{array}$ \\
\hline Referências & $\begin{array}{l}\text { Page-Cutrara K. Prebriefing in nursing simulation: a concept analysis. Clin Simul } \\
\text { Nurs. 2015; 11(7):335-340. doi: doi.org/10.1016/j.ecns.2015.05.001 } \\
\text { Gantt LT. The effect of preparation on anxiety and performance in summative } \\
\text { simulations Clin Simul Nurs. 2013; 9:25-33. doi: 10.1016/j.ecns.2011.07.004 } \\
\text { International Nursing Association for Clinical Simulation and Learning } \\
\text { (INACSL). Standards of best practice [Internet]. } 2016 \text { [acesso } 2020 \text { Jun 26]. } \\
\text { Disponível em: https://www.inacsl.org/inacsl-standards-of-best-practice- } \\
\text { simulation/ } \\
\text { Tyerman J, Flude ML, Graham L, Coffey S, Lynch EO. A systematic review of } \\
\text { health care presimulation preparation and briefing effectiveness. Clin Simul Nurs. } \\
\text { 2019; } 27: 12-25,2019 \text {. doi:10.1016/j.ecns.2018.11.002 } \\
\text { Tyerman J, Luctkar M, Graham L, Coffey S, Lynch E. O Pre-simulation preparation and } \\
\text { briefing practices for healthcare professionals and students: a systematic review } \\
\text { protocol. Joanna Briggs Institute. } 2016 ; 14(8): 80-9 . \text { doi:10.11124/JBISRIR-2016-003055 }\end{array}$ \\
\hline
\end{tabular}

\section{Referências}

1. McEnroe-Petitte D, Farris C. Using gaming as an active teaching strategy in nursing education. Teach Learn Nurs. 2020;15(1):61-5. doi: 10.1016/j.teln.2019.09.002

2. Lamé G, Dixon-Woods M. Using clinical simulation to study how to improve quality and safety in healthcare. BMJ Simul Technol Enhanc Learn. 2020;6(2):87-94. doi: 10.1136/bmjstel-2018-000370

3. Inglis S, Nelson L. Exploring the effects of clinical simulation on nursing students' learning and practice. Ment Health Pract. 2020;23(5):38-42. doi: https://doi.org/10.7748/mhp.2020.e1355

4. Nascimento JSG, Costa ABF, Sangiovani JC, Silva TCS, Regino DSG, Dalri MCB. Pré-simulação, prébriefing ou briefing na simulação em enfermagem: quais as diferenças? Rev Eletrônica Enferm [Internet]. 2020 [cited 2020 Nov 12];22:60171. Available from: https://revistas.ufg.br/fen/article/view/60171

5. Tyerman J, Lucktar-Flude M, Graham L, Coffey S, Olsen-Lynch E. A systematic review of health care presimulation preparation and briefing effectiveness. Clin Simul Nurs. 2019;27:12-25. doi: 
Simulação clínica: construção e validação de roteiro para o Suporte Básico de Vida no adulto | 24

10.1016/j.ecns.2018.11.002

6. Hansen CM, Rosenkranz SM, Folke F, Zinckernagel L, Tjørnhøj-Thomsen T, Torp-Pederson C, et al. Lay bystanders' perspectives on what facilitates cardiopulmonary resuscitation and use of automated external defibrillators in real cardiac arrests. J Am Heart Assoc. 2017;6(3):e004572. doi: https://doi.org/10.1161/JAHA.116.004572

7. Page-Cutrara K, Turk M. Impact of prebriefing on competency performance, clinical judgment and experience in simulation: an experimental study. Nurse Educ Today. 2017;48:78-83. doi: 10.1016/j.nedt.2016.09.012

8. Leigh G, Steuben F. Setting learners up for success: presimulation and prebriefing strategies. Teach Learn Nurs. 2018;13(3):185-9. doi: https://doi.org/10.1016/j.teln.2018.03.004

9. Roh YS, Ahn JW, Kim E, Kim J. Effects of prebriefing on psychological safety and learning outcomes. Clin Simul Nurs. 2018;25:12-9. doi: https://doi.org/10.1016/j.ecns.2018.10.001

10. Alves MG, Pereira VOS, Batista DF, Cordeiro ALP, Nascimento JSG, Dalri MCB. Construction and validation of a questionnaire for cardiopulmonary resuscitation knowledge assessment. Cogitare Enferm. 2019;24:e64560. doi: http://dx.doi.org/10.5380/ce.v24i0.64560

11. Pasquali L. Instrumentação psicológica: fundamentos e práticas. Porto Alegre: Artmed; 2009.

12. American Heart Association (AHA). Destaques das diretrizes de RCP e ACE de 2020 da American Heart Association [Internet]. Dallas: American Heart Association ; 2020 [cited 2020 Nov 12]. Available from: https://cpr.heart.org/-/media/cpr-files/cpr-guidelines- files/highlights/hghlghts_2020eccguidelines_portuguese.pdf

13. Whittemore R, Knafl K. The integrative review: updated methodology. J Adv Nurs. 2005;52(5):546-53. doi: https://doi.org/10.1111/j.1365-2648.2005.03621.x

14. Alarcón-Gil MT, Osorio Toro S, Baena Caldas GP. The evidence-based medicine PICO strategy applied to dentistry using MeSH, Emtree and DeCS. Rev Fac Odontol Univ Antioq. 2019;31(1):91-101. doi: https://doi.org/10.17533/udea.rfo.v31n1-2a8

15. Ouzzani M, Hammady H, Fedorowicz Z, Elmagarmid A. Rayyan: a web and mobile app for systematic reviews. Syst Rev. 2016;5(1):210. doi: https://doi.org/10.1186/s13643-016-0384-4

16. Ursi ES, Galvão CM. Prevenção de lesões de pele no perioperatório: revisão integrativa da literatura. Rev Latinoam Enferm [Internet]. 2006 [acesso em 2020 nov 12];14(1):124-31. Disponível em: http://www.scielo.br/pdf/rlae/v14n1/v14n1a17

17. Melnyk BM, Fineout-Overholt E. Evidence-based practice in nursing \& healthcare: a guide to best practice. 2nd ed. Philadelphia: Wolters Kluwer Health; Lippincott Williams \& Wilkins; 2019.

18. Minayo MCS. Amostragem e saturação em pesquisa qualitativa: consensos e controvérsias. Rev 
Pesqui Qual [Internet]. 2017 [acesso em 2020 nov 12];5(7):1-12. Disponível em: https://editora.sepq.org.br/index.php/rpq/article/view/82/59

19. Tricco AC, Lillie E, Zarin W, O'Brien KK, Colquhoun H, Levac D. PRISMA Extension for scoping reviews (PRISMA-ScR): checklist and explanation. Ann Intern Med. 2018;169(7):467-73. doi: https://doi.org/10.7326/M18-0850

20. Santos APFB, Andrade JF, Alves GCS, Silva SD, Sanches C, Chequer FMD. Analysis of the use of the Delphi technique for decision-making in critically ill patients: a systematic review. Rev Med [Internet]. 2020 [acesso em 2020 nov 12];99(3):291-304. Disponível em: https://www.revistas.usp.br/revistadc/article/view/160704/161326

21. Fehring RJ. The Fehring model. In: Carrol-Johnson RM, Paquete M. Classification of nursing diagnoses: proceeding of the tenth conference. Philadelphia: Lippincott Company; 1994. p. 55-62.

22. Vakili MM, Jahangiri N. Content validity and reliability of the measurement tools in educational behavioral, and health sciences research. J Med Educ Dev. 2018;10(28):106-19. doi: https://doi.org/10.29252/edcj.10.28.106

23. Silveira MB, Saldanha RP, Leite JCC, Silva TOF, Silva T, Filippin LI. Construção e validade de conteúdo de um instrumento para avaliação de quedas em idosos. Einstein. 2018;16(2):eAO4154. doi: https://doi.org/10.1590/s1679-45082018ao4154

24. Steinemann S, Bhatt A, Suares G, Wei A, Ho N, Kurosawa G, et al. Trauma team discord and the role of briefing. J Trauma Acute Care Surg. 2016;81(1):184-9. doi: https://doi.org/10.1097/TA.0000000000001024

25. Roh YS, Ahn JW, Kim E, Kim J. Effects of prebriefing on psychological safety and learning outcomes. Clin Simul Nurs. 2018;25:12-9. doi: https://doi.org/10.1016/j.ecns.2018.10.001

26. Kim YJ, Noh GO, Im YS. Effect of step-based prebriefing activities on flow and clinical competency of nursing students in simulation-based education. Clin Simul Nurs. 2017;13(11):544-51. doi: https://doi.org/10.1016/j.ecns.2017.06.005

27. Chamberlain J. The impact of simulation prebriefing on perceptions of overall effectiveness, learning, and self-confidence in nursing students. Nurs Educ Perspect. 2017;38(3):119-25. doi: https://doi.org/10.1097/01.NEP.0000000000000162

28. Coram C. Expert role modeling effect on novice nursing students' clinical judgment. Clin Simul Nurs. 2016;12(9):385-91. doi: https://doi.org/10.1016/j.ecns.2016.04.009

29. Franklin AE, Sideras S, Gubrud-Howe P, Lee CS. Comparison of expert modeling versus voice-over powerpoint lecture and presimulation readings on novice nurses' competence of providing care to multiple patients. J Nurs Educ. 2014;53(11):615-22. doi: https://doi.org/10.3928/01484834-20141023-01 
Simulação clínica: construção e validação de roteiro para o Suporte Básico de Vida no adulto | 26

30. Beverly JD. Interactive pre-simulation strategies: engaging students in experimental learning from the start. J Syst Cybern Inf [Internet]. 2014 [cited 2020 Nov 12];12(1):69-75. Disponível em: http://www.iiisci.org/journal/cr\$/sci/pdfs/fa223qu13.pdf

Editora Científica: Tânia Solange Bosi de Souza Magnago

Editora associada: Rhanna Emanuela Fontenele Lima de Carvalho

\section{Autor correspondente}

Juliana da Silva Garcia Nascimento

E-mail: mestradounesp28@yahoo.com.br

Endereço: Rua Osório Joaquim Guimarães, no 281. Ap.203, Bloco 05. Parque São Geraldo. Uberaba- Minas Gerais.

CEP: 38030130

\section{Contribuições de Autoria}

\section{1 - Juliana da Silva Garcia Nascimento}

Concepção ou desenho do estudo/pesquisa, análise e/ou interpretação dos dados, revisão final com participação crítica e intelectual no manuscrito.

\section{2 - Kleiton Gonçalves do Nascimento}

Concepção ou desenho do estudo/pesquisa, análise e/ou interpretação dos dados, revisão final com participação crítica e intelectual no manuscrito.

\section{3 - Daniela da Silva Garcia Regino}

Concepção ou desenho do estudo/pesquisa, análise e/ou interpretação dos dados.

\section{4 - Mateus Goulart Alves}

Concepção ou desenho do estudo/pesquisa, análise e/ou interpretação dos dados.

\section{5 - Jordana Luiza Gouvêa de Oliveira}

Concepção ou desenho do estudo/pesquisa, análise e/ou interpretação dos dados.

\section{6 - Maria Celia Barcellos Dalri}

Concepção ou desenho do estudo/pesquisa, revisão final com participação crítica e intelectual no manuscrito.

\section{Como citar este artigo}

Nascimento JSG, Nascimento KG, Regino DSG, Alves MG, Oliveira JLG, Dalri MCB. Simulação clínica: construção e validação de roteiro para o Suporte Básico de Vida no adulto. Rev. Enferm. UFSM. 2021 [Acesso em: Ano Mês Dia]; vol.11 e44: 1-26 DOI:https://doi.org/10.5902/2179769254578 